April 2, 2001

LBNL-47684

Revised October 23, 2001 (for PRL length limit)

hep-ph/0104024

\title{
The $Z \rightarrow \bar{b} b$ decay asymmetry: lose-lose for the Standard Model 1
}

\author{
Michael S. Chanowitय' \\ Theoretical Physics Group \\ Ernest Orlando Lawrence Berkeley National Laboratory \\ University of California \\ Berkeley, California 94720
}

\begin{abstract}
Combining precision measurements and the Higgs boson search limit, the electroweak data has evolved to a point where new physics is favored whether the $3.2 \sigma$ $A_{F B}^{b}$ anomaly is genuine or not. Such new physics could greatly alter the inferred value of the Higgs boson mass.
\end{abstract}

\footnotetext{
${ }^{1}$ This work is supported in part by the Director, Office of Science, Office of High Energy and Nuclear Physics, Division of High Energy Physics, of the U.S. Department of Energy under Contract DE-AC0376SF00098

${ }^{2}$ Email: chanowitz@lbl.gov
} 


\section{Disclaimer}

This document was prepared as an account of work sponsored by the United States Government. While this document is believed to contain correct information, neither the United States Government nor any agency thereof, nor The Regents of the University of California, nor any of their employees, makes any warranty, express or implied, or assumes any legal liability or responsibility for the accuracy, completeness, or usefulness of any information, apparatus, product, or process disclosed, or represents that its use would not infringe privately owned rights. Reference herein to any specific commercial products process, or service by its trade name, trademark, manufacturer, or otherwise, does not necessarily constitute or imply its endorsement, recommendation, or favoring by the United States Government or any agency thereof, or The Regents of the University of California. The views and opinions of authors expressed herein do not necessarily state or reflect those of the United States Government or any agency thereof, or The Regents of the University of California.

Lawrence Berkeley National Laboratory is an equal opportunity employer. 
Introduction - A decade of beautiful experiments have provided increasingly precise tests of the Standard Model (SM) of elementary particle physics. The data tests the SM, probes for new physics, and is sensitive to $m_{H}$, the mass of the still undiscovered Higgs boson which gives mass to the elementary particles. Currently it implies an upper bound on $m_{H}$ of order $200 \mathrm{GeV}$, while direct searches have established a lower limit of $113.5 \mathrm{GeV}$. [1]

In the most recent analysis of the electroweak data the $Z \rightarrow \bar{b} b$ front-back asymmetry, $A_{F B}^{b}$, differs by $3.2 \sigma(99.9 \% \mathrm{CL})$ from the SM fit.[1] It could represent new physics, but a few red flags suggest caution: (1) the direct determination of $A_{b}$ from the front-back left-right asymmetry, $A_{F B L R}^{b}$, is consistent with the SM $(0.7 \sigma)$ while $A_{b}$ extracted from $A_{b}=4 A_{F B}^{b} / 3 A_{l}$ (where $A_{l}$ is the leptonic asymmetry) disagrees by $3.5 \sigma(99.95 \% \mathrm{CL}),(2)$ $Z \rightarrow \bar{b} b$ measurements have proven notoriously difficult in the past, and (3) there is no hint of an $R_{b}$ anomaly to match the $A_{b}$ anomaly, requiring a degree of tuning of the left and right-handed $Z \bar{b} b$ couplings with an extremely large shift in the right-handed coupling.

The situation is then quite puzzling. The result could be a statistical fluctuation, but statistical criteria reviewed below tell us this is very unlikely. The remaining two possibilities are new physics or subtle systematic error. While great care and effort have been focused on understanding and reducing the systematic uncertainties, further work is needed before we can choose clearly between the two possibilities. If the explanation is systematic error and $A_{F B}^{b}$ is omitted, the global SM fit, which is poor with $A_{F B}^{b}$ included, becomes excellent, but the predicted value of $m_{H}$, the Higgs boson mass, falls to low values in conflict with the direct search limit, $m_{H}>113.5$ at $95 \%$ CL. pl To remove the inconsistency new physics would be required to modify the predictions based on the radiative corrections. New physics is then favored whether $A_{F B}^{b}$ is affected by systematic error or not, and $m_{H}$ cannot be predicted without disentangling the effect of the new physics.

Though less precise it is striking that the values of $x_{W}^{l}=\sin ^{2} \theta_{W}^{l}$, the effective leptonic mixing angle, extracted from the two other hadronic asymmetry measurements, $A_{F B}^{c}$ and $Q_{F B}$, agree with $x_{W}^{l}$ from $A_{F B}^{b}$ and deviate from the SM fit. Combined the three measurements differ from the SM fit at $99.5 \% \mathrm{CL}$. At the same time they are the only precision measurements that raise the predicted value of $m_{H}$ toward the range required by the direct search limit. We show below that all other $m_{H}$-sensitive precision measurements favor values much lower than $113.5 \mathrm{GeV}$. The set of measurements that are consistent with the global fit are inconsistent with the search limit while the measurements that are essential for consistency with the search limit are inconsistent with the global fit.

\footnotetext{
${ }^{3}$ N.B., the $95 \%$ lower limit does not imply a $5 \%$ chance that the Higgs boson is lighter than $113.5 \mathrm{GeV}$; rather it means that if the Higgs mass were actually $113.5 \mathrm{GeV}$ there would be a $5 \%$ chance for it to have escaped detection. The likelihood for $m_{H}<113.5 \mathrm{GeV}$ from the direct searches is much smaller than $5 \%$. See for instance the discussion in section 5 of [2].
} 
The data-In the latest data the $3.5 \sigma$ difference in the SM determinations of $x_{W}^{l}$ from $A_{L R}$ and $A_{F B}^{b}$ drives a poor fit of the 7 asymmetries used to determine $x_{W}^{l}$, with $\chi^{2} / d o f=15.5 / 6$ and $\mathrm{CL}=0.013$. The four leptonic measurements, $A_{L R}, A_{F B}^{l}, A_{e}, A_{\tau}$, agree very well with one another, $\chi^{2} / d o f=2.7 / 3$, as do the three hadronic determinations from $A_{F B}^{b}, A_{F B}^{c}, Q_{F B}, \chi^{2} / d o f=0.1 / 2$, while the aggregated leptonic and hadronic determinations of $x_{W}^{l}$ differ by $3.6 \sigma(99.97 \% \mathrm{CL})$.

The four leptonic asymmetries provide the first, third, fourth, and fifth most precise of the 7 determinations of $x_{W}^{l}$. Because they are consistent, large systematic errors would have to conspire to affect each measurement in a similar way, which is very unlikely because they are measured by three very different methods. The same cannot be said of the hadronic asymmetries, which share common systematic issues. All three hadronic measurements require similar QCD corrections and make common use of fragmentation and decay models. As in the $R_{b}$ anomaly, $\bar{b} b$ and $\bar{c} c$ events constitute backgrounds for one another.f

In the most recent analysis the $A_{F B}^{b}$ and $A_{F B}^{c}$ measurements are assigned a $16 \%$ correlation $[1]$.

Taking a wider perspective, it is useful to consider the 15 degrees of freedom in the global $\mathrm{SM}$ fit of all data reported in reference [1]. Even in that framework a $\geq 3.2 \sigma$ discrepancy is very unlikely, with probability $1-0.9986^{15}=0.021$. As noted above, $A_{F B}^{b}$ also drives the poor $\chi^{2}$ of that fit, $\chi^{2} / d o f=26 / 15$ and $\mathrm{CL}=0.038$. With the contribution of $A_{F B}^{b}$ removed the same fit parameters yield $\chi^{2} / d o f=15.8 / 14$ corresponding to a robust $\mathrm{CL}=0.33$. If instead the second most deviant measurement, $A_{L R}$, is omitted, the improvement is much smaller, with $\chi^{2} / \operatorname{dof}=23.2 / 14$ and $\mathrm{CL}=0.057$.

Another feature of the data also points to $A_{F B}^{b}$ as the 'odd man out.' The $Z \bar{b} b$ vertex factor, $A_{b}$, is predicted very precisely in the SM and agrees well $(0.7 \sigma)$ with the direct determination at the SLC from $A_{F B L R}^{b}$. But the determination from $A_{b}=4 A_{F B}^{b} / 3 A_{l}$ disagrees with the SM by $3.5 \sigma$ and from the directly measured $A_{F B L R}^{b}$ by $1.8 \sigma$.

The evidence for new physics in the $Z \bar{b} b$ vertex is compelling on a purely statistical level, and the third generation quarks are a plausible venue for new physics connected to the symmetry breaking sector. But the disagreement with $A_{F B L R}^{b}$ and the past history of $Z \rightarrow \bar{b} b$ measurements suggest caution. While the lessons of the $R_{b}$ anomaly have been refined and applied to $A_{F B}^{b}$, the latter measurement involves additional subtleties. Systematic error could in principle provide an escape path for the SM. But we will see in the next section that the path is rather narrow if it is open at all.

Results - In this section we present $\chi^{2}$ fits of $m_{H}$ and compare them with the search

\footnotetext{
${ }^{4}$ It is suggestive that the signs of both the $A_{F B}^{b}$ and $A_{F B}^{c}$ anomalies are as would be expected if $c$ 's were misidentified as $\bar{b}$ 's and vice-versa, although the systematic error currently budgeted to this effect is much smaller [1] than the anomalies.
} 
limit. To confront the predictions of the SM as directly as possible we focus on the directly measured, $m_{H}$-sensitive observables. The observables with the greatest impact are $x_{W}^{l}$ and $m_{W}$. The other directly measured, $m_{H}$-sensitive $Z$-pole observables are the total width $\Gamma_{Z}=2.4952(23) \mathrm{GeV}$ and the ratio of hadronic to leptonic partial widths, $R_{l}=\Gamma_{h} / \Gamma_{l}=$ 20.767(25). [1] For $m_{Z}, m_{W}$ and $m_{t}$ we use the directly measured values, currently $m_{Z}=$ 91.1875(21) $\mathrm{GeV}, m_{W}=80.448(34) \mathrm{GeV}$ and $m_{t}=174.3(5.1) \mathrm{GeV}$. [1]

The strong coupling is taken to be $\alpha_{S}\left(m_{Z}\right)=0.118(3)$. The greatest parametric uncertainty is from the electromagnetic coupling at the $Z$ pole, $\alpha\left(m_{Z}\right)$, in particular from $\Delta \alpha_{5}$, the five flavor hadronic contribution to $\Delta \alpha$, which renormalizes $\alpha$ by $\alpha\left(m_{Z}\right)=\alpha(0) /(1-\Delta \alpha)$. We use five determinations: two experiment-driven [3, t] based on the most recent data and three theory-driven. 5, 6, 调 Gaussian errors are assumed for all experimental quantities. We use the two loop radiative correction package from ZFITTER \&], version 6.30, to compute the SM values of the four observables as a function of $m_{H}$ and the four experimentally determined parameters, $m_{Z}, m_{t}, \alpha\left(m_{Z}\right)$, and $\alpha_{S}\left(m_{Z}\right)$. Taking as inputs the all-data fit values [1] for $m_{Z}, m_{t}, \Delta \alpha_{5}, \alpha_{S}$, and $m_{H}$, we reproduce the results (from ZFITTER v6.35) in [1] as follows: $x_{W}^{l}: 0.23142 / 0.23142, m_{W}: 80.394 / 80.393, \Gamma_{Z}: 2.4960 / 2.4962$, and $R_{l}: 20.737 / 20.740$. The effect of such differences on $\chi^{2}$ is negligible.

For two reasons we first consider just $m_{W}$ and the $Z$-pole measurements, $\Gamma_{Z}$ and $R_{l}$ : (1) they are not affected by the issues affecting the asymmetries and (2) the determination of $m_{H}$ from them is less sensitive to the uncertainty from $\alpha\left(m_{Z}\right)$. The results are striking. Figure 1 shows $\Delta \chi^{2}=\chi^{2}-\chi_{\min }^{2}$ as a function of $m_{H}$ obtained from $m_{W}$ alone and in combination with $\Gamma_{Z}$ and $R_{l}$. At each value of $m_{H}$ the experimental parameters $m_{t}, \alpha\left(m_{Z}\right)$, and $\alpha_{S}\left(m_{Z}\right)$ are chosen to minimize the sum of the $\chi^{2}$ contributions from $m_{W}, m_{t}, \alpha\left(m_{Z}\right)$, and $\alpha_{S}$ (and also from $\Gamma_{Z}$ and $R_{l}$ in the case of the second fit). The results are summarized in table 1 for the five choices of $\alpha\left(m_{Z}\right)$. For the fit based just on $m_{W}$ the central value of $m_{H}$ falls between 21 and $28 \mathrm{GeV}$, with $m_{H}<113.5 \mathrm{GeV}$ favored at 94 to $92 \% \mathrm{CL}$. For the fit with $\Gamma_{Z}$ and $R_{l}$ included, the results are even less sensitive to $\alpha\left(m_{Z}\right)$ and are shifted to lower $m_{H}$, 15 - $17 \mathrm{GeV}$, with $\mathrm{CL}\left(m_{H}<113.5 \mathrm{GeV}\right)$ increased to between 98 and $97 \%$.

We next consider the effect of the asymmetry measurements in the framework of the hypothesis that the $A_{F B}^{b}$ anomaly results from undetected systematic error. As discussed above, $A_{F B}^{b}, A_{F B}^{c}$ and $Q_{F B}$ share common sytematics so that the most reliable determination of $x_{W}^{l}$ would in this case be provided by the four leptonic asymmetry measurements, which are very unlikely to have common systematic uncertainties. Results based on the leptonic asymmetries, which yield $x_{W}^{l}=0.23113(20)$, combined with $m_{W}, \Gamma_{Z}$, and $R_{l}$ are shown in

\footnotetext{
5 The older theory-driven determinations [5, 6] are included because they are consistent with the new data.

${ }^{6}$ We have checked that varying $m_{Z}$ has negligible effect on $\chi^{2}$.
} 
figure 2 and summarized in table 2 where they are labeled ' $+L 4$.' The central values are in the range $27-44 \mathrm{GeV}$, with $\mathrm{CL}\left(m_{H}<113.5 \mathrm{GeV}\right)$ from 98 to $94 \%$. As in table 1 there is a significant conflict with the search limit, though with more dependence on $\alpha\left(m_{Z}\right)$.

For completeness we also consider the effect of the two lower precision hadronic asymmetry measurements, combining $Q_{F B}\left(x_{W}^{l}=0.23118(20)\right)$ and $A_{F B}^{c}\left(x_{W}^{l}=0.23127\right.$ (19)) sequentially with the previously considered observables. The results, in figure 2 and table 2, conflict with the search limit, though less decisively. Finally we exhibit the results with all seven asymmetry measurements included, denoted ' $+A_{F B}^{b}$ ' in table 2 , with $x_{W}^{l}=0.23156$ (17). As for the usual global SM fits, $m_{H}$ is centered around $100 \mathrm{GeV}$ and the fits are consistent with the search limit for all $\alpha\left(m_{Z}\right)$.

To summarize, each fit with $A_{F B}^{b}$ omitted is in conflict with the search limit, and the fits based on the data that is most reliable if the $A_{F B}^{b}$ anomaly is a systematic effect - table 1 and the ' $+L 4$ ' fit of table 2 - have the most significant conflicts.

Discussion-The greatest source of uncertainty is the sensitivity to $\alpha\left(m_{Z}\right)$ of the fits that include asymmetry data, which makes the lack of asymmetry data in table 1 especially interesting. The theoretical uncertainty from uncalculated diagrams is smaller than from $\alpha\left(m_{Z}\right)$, as can be seen in figure 13 of [1] where the "blue band" estimating the theoretical uncertainty is less than the difference resulting from $\alpha\left(m_{Z}\right)$ for [4] compared to [7]. The figure also shows that the prediction of ZFITTER as employed in [1] lies near the large- $m_{H}$ edge of the blue band. Since, as noted above, our ZFITTER calculations closely reproduce those of [1], our estimates of the conflict with the search limits are conservative relative to the other libraries/settings used to generate the blue band.

We have found that new physics is favored by the data whether the $A_{F B}^{b}$ anomaly reflects new physics or systematic error. An important consequence is that the evidence from the SM fit favoring a light Higgs boson becomes less credible. It can be maintained only if the $A_{F B}^{b}$ anomaly turns out to be a statistical fluctuation.] The most generous estimate of the likelihood of this possibility is the poor $3.8 \% \chi^{2}$ CL of the global SM fit, [1], which is due almost entirely to the deviation of $A_{F B}^{b}$ from the fit as noted above. Otherwise, whether the anomaly is a genuine signal of new physics or a systematic artifact, $A_{F B}^{b}$ cannot be used to determine $x_{W}^{l}$, and the resulting conflict with the search limit favors new physics contributions to remove the contradiction.

We can get a rough idea of the new physics contributions that would be needed by considering just $x_{W}^{l}$ and $m_{W}$, using the deviation from the SM for any given value of $m_{H}$, $\delta x_{W}^{l}$ and $\delta m_{W}$, to compute the corresponding oblique parameters 9] $S$ and $T$. Taking $x_{W}^{l}$

\footnotetext{
${ }^{7}$ If the discrepancies in the SM fit are statistical fluctuations, the appropriate fits are those in the bottom line of Table 2 , and the measurements of all the $m_{H}$-sensitive observables, not just $A_{F B}^{b}$, must have fluctuated significantly from their true values.
} 
from the 4 leptonic asymmetries and using the direct measurement of $m_{W}$, we find, e.g., for $m_{H}=114,300,1000,2000 \mathrm{GeV}$ that the corresponding values are $(S, T)=(-0.02,0.16)$, $(-0.08,0.27),(-0.11,0.48),(-0.09,0.65)$, where $m_{H}=2000 \mathrm{GeV}$ is a 'stand-in' for dynamical symmetry breaking. The existing data cannot choose among these possibilities.

The unexpected emergence of evidence for new physics at the end of the LEP/SLC decade is a cautionary signal to keep an open mind as to the ultimate explanation. If the $A_{F B}^{b}$ anomaly is genuine, it signals new physics not anticipated by popular theoretical models. If the anomaly is genuine and unique to the third generation, it will also affect $\bar{b} s$, $\bar{b} d$, and $\bar{s} d$ neutral currents via non-SM $Z$ penguin amplitudes, though the precise effects are not readily predicted. If the anomaly is not genuine, the conflict with the search limit is for now our only evidence of the new physics and we are left with even fewer clues as to its nature.

The evidence for new physics presented here may be weakened or strengthened by future measurements, not only of $A_{F B}^{b}$ and the other asymmetries but also of $m_{W}$ and $m_{t}$. New facilities will be needed to answer the questions posed by the current data, including a second generation $Z$ factory. Better measurements of $R_{e^{+} e^{-}}$would be needed to determine $\alpha\left(m_{Z}\right)$ with enough precision to realize the potential precision of a new $Z$ factory for $x_{W}^{l}$. [3] This will be important even after the Higgs sector is discovered, since precise comparisons of the electroweak data with predictions based on the observed Higgs sector will provide invaluable guidance on whether additional new physics exists at yet higher scales. The evidence of the present data for unspecified new physics underscores the importance of framing the search for the Higgs sector in the most general form.

Note added-Data presented after this work was completed[10] differs slightly from the data considered above: $A_{F B}^{b}$ disagrees with the SM fit by $2.9 \sigma(\mathrm{CL}=99.6 \%)$, and the leptonic and hadronic SM determinations of $x_{W}^{l}$ disagree by $3.3 \sigma(\mathrm{CL}=99.9 \%)$. The likelihood of the statistical fluctuation hypothesis increases to a still small probability, e.g., from $3.8 \%$ to $6.7 \%$ as gauged by the global fit. The analysis of the systematic error hypothesis is unaffected since the fits which omit $A_{F B}^{b}$ change very little, and the contradiction with the search limit persists at the levels quoted above.

Acknowledgements I am grateful to A. Höcker, P. Rowson, and R. Cahn for very helpful discussions. I also wish to thank T. Abe, D. Bardin, M. Grünewald, and G. Passarino for useful correspondence and $\mathrm{H}$. Chanowitz for computing facilities. 


\section{References}

[1] The LEP Collaborations ALEPH, DELPHI, L3, OPAL, the LEP Electroweak Working Group, and the SLD Heavy Flavour and Electroweak Groups, LEPEWWG/2001-01, May 2001.

[2] M. Chanowitz, Phys.Rev.D59:073005,1999, e-Print hep-ph/9807452.

[3] F. Jegerlehner, hep-ph/0104304, Apr 2001.

[4] H. Burkhardt and B. Pietrzyk, LAPP-EXP-2001-03, Feb 2001 (submitted to Phys. Lett. $B)$.

[5] M. Davier and A. Höcker, Phys. Lett. B435:427,1998.

[6] J. Kühn and M. Steinhauser, Phys. Lett. B437:425,1998.

[7] A.D. Martin, J. Outhwaite, M.G. Ryskin, IPPP-00-15, DTP-00-84, Dec 2000, e-Print hep-ph/0012231.

[8] D. Bardin et al., Comput.Phys.Commun.133:229,2001, e-Print hep-ph/9908433.

[9] M. Peskin and T. Takeuchi, Phys. Rev. D46:381,1992.

[10] S. Mele, presented at EPS-HEP 2001, Budapest, July 12-18, 2001, to be published in the proceedings. 
Table 1. Fit results without asymmetry measurements for five determinations of $\alpha\left(M_{Z}\right)$. For each fit the central value of $m_{H}$ is shown in $\mathrm{GeV}$ and the confidence level (CL) for $m_{H}>113.5$ $\mathrm{GeV}$. Results for $m_{W}$ alone are shown in the first two rows, and for the combination of $m_{W}$, $\Gamma_{Z}$, and $R_{l}$ in the last two.

\begin{tabular}{c|c|ccccc} 
& & $\mathrm{J}[3]$ & $\mathrm{BP}[\mathbf{4}$ & $\mathrm{MOR}[\mathbf{7}]$ & $\mathrm{DH}[5]$ & $\mathrm{KS}[\mathrm{G}]$ \\
& $\Delta \alpha_{5}$ & $0.027896(395)$ & $0.02761(36)$ & $0.027426(190)$ & $0.02763(16)$ & $0.02775(17)$ \\
\hline \hline$m_{W}$ & $m_{H}$ & 21 & 26 & 28 & 25 & 23 \\
& $\mathrm{CL}$ & 0.057 & 0.071 & 0.080 & 0.068 & 0.062 \\
\hline$+\Gamma_{Z}, R_{l}$ & $m_{H}$ & 15 & 17 & 15 & 17 & 15 \\
& $\mathrm{CL}$ & 0.023 & 0.028 & 0.032 & 0.027 & 0.024 \\
\hline \hline
\end{tabular}

Table 2. Fit results with asymmetry measurements included. The format is as in table 1. The first fit reflects the combination of $m_{W}, \Gamma_{Z}$, and $R_{l}$ together with the four leptonic asymmetry measurements, ' $L 4$ '. Successive fits incrementally include $Q_{F B}, A_{F B}^{c}$, and $A_{F B}^{b}$.

\begin{tabular}{c|c|ccccc} 
& & $\mathrm{J}[3]$ & $\mathrm{BP}[4$ & $\mathrm{MOR}[\mathbf{0}]$ & $\mathrm{DH}[5]$ & $\mathrm{KS}[6]$ \\
& $\Delta \alpha_{5}$ & $0.027896(395)$ & $0.02761(36)$ & $0.027426(190)$ & $0.02763(16)$ & $0.02775(17)$ \\
\hline \hline$+L 4$ & $m_{H}$ & 27 & 37 & 44 & 39 & 34 \\
& $\mathrm{CL}$ & 0.019 & 0.041 & 0.060 & 0.033 & 0.023 \\
\hline$+Q_{F B}$ & $m_{H}$ & 33 & 43 & 57 & 46 & 39 \\
& $\mathrm{CL}$ & 0.028 & 0.058 & 0.087 & 0.049 & 0.034 \\
\hline$+A_{F B}^{c}$ & $m_{H}$ & 43 & 53 & 61 & 61 & 55 \\
& $\mathrm{CL}$ & 0.053 & 0.10 & 0.14 & 0.091 & 0.069 \\
\hline$+A_{F B}^{b}$ & $m_{H}$ & 86 & 110 & 114 & 102 & 89 \\
& $\mathrm{CL}$ & 0.26 & 0.36 & 0.50 & 0.40 & 0.38 \\
\hline \hline
\end{tabular}




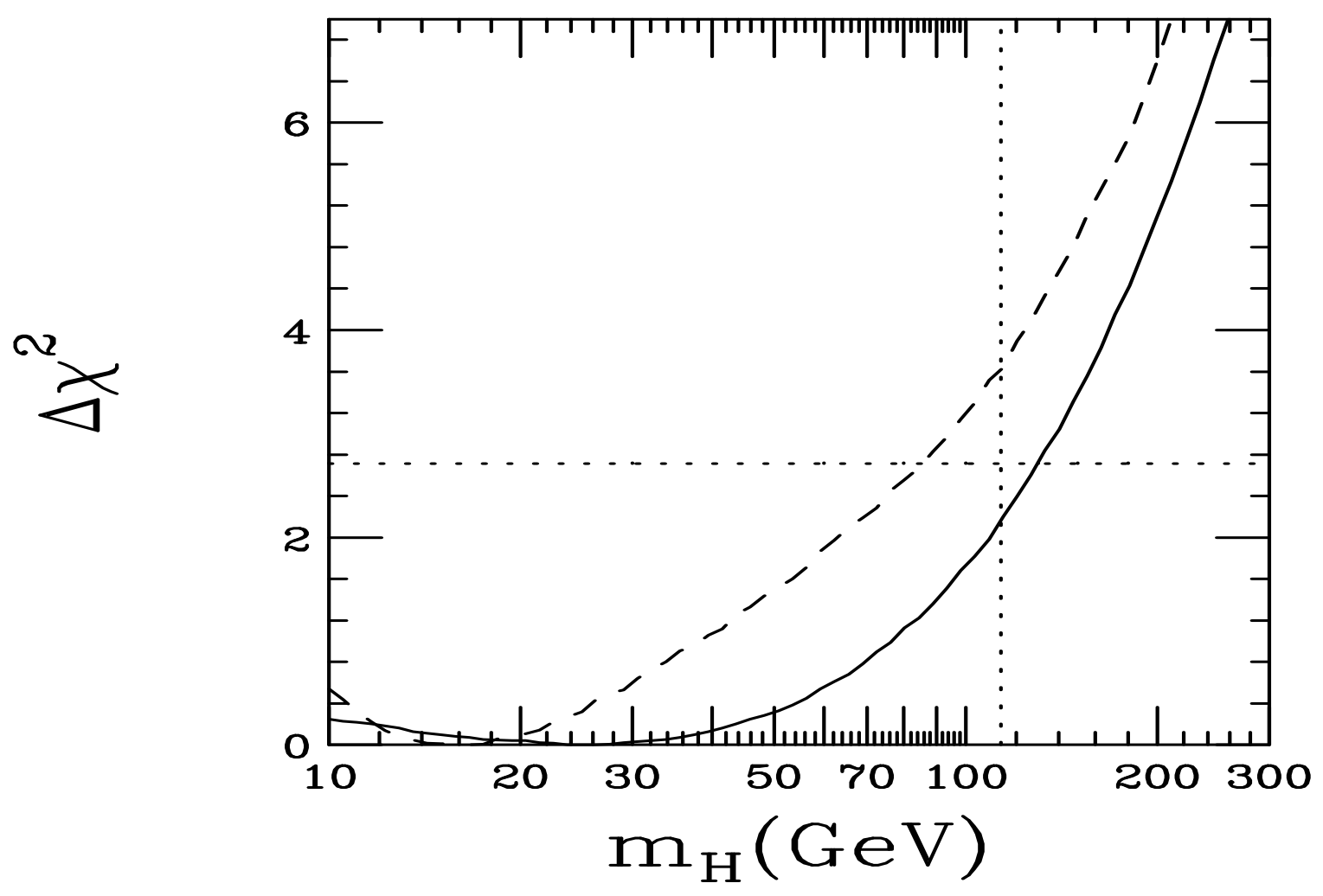

Figure 1: $\chi^{2}$ distributions as a function of $m_{H}$. The solid line is obtained from $m_{W}$ alone and the dashed line from the combined fit of $m_{W}, \Gamma_{Z}$, and $R_{l}$. The vertical dotted line indicates the direct search lower limit and the horizontal dotted line indicates the value of $\Delta \chi^{2}$ corresponding to a $95 \% \mathrm{CL}$ upper limit. $\alpha\left(m_{Z}\right)$ is from [4]. 


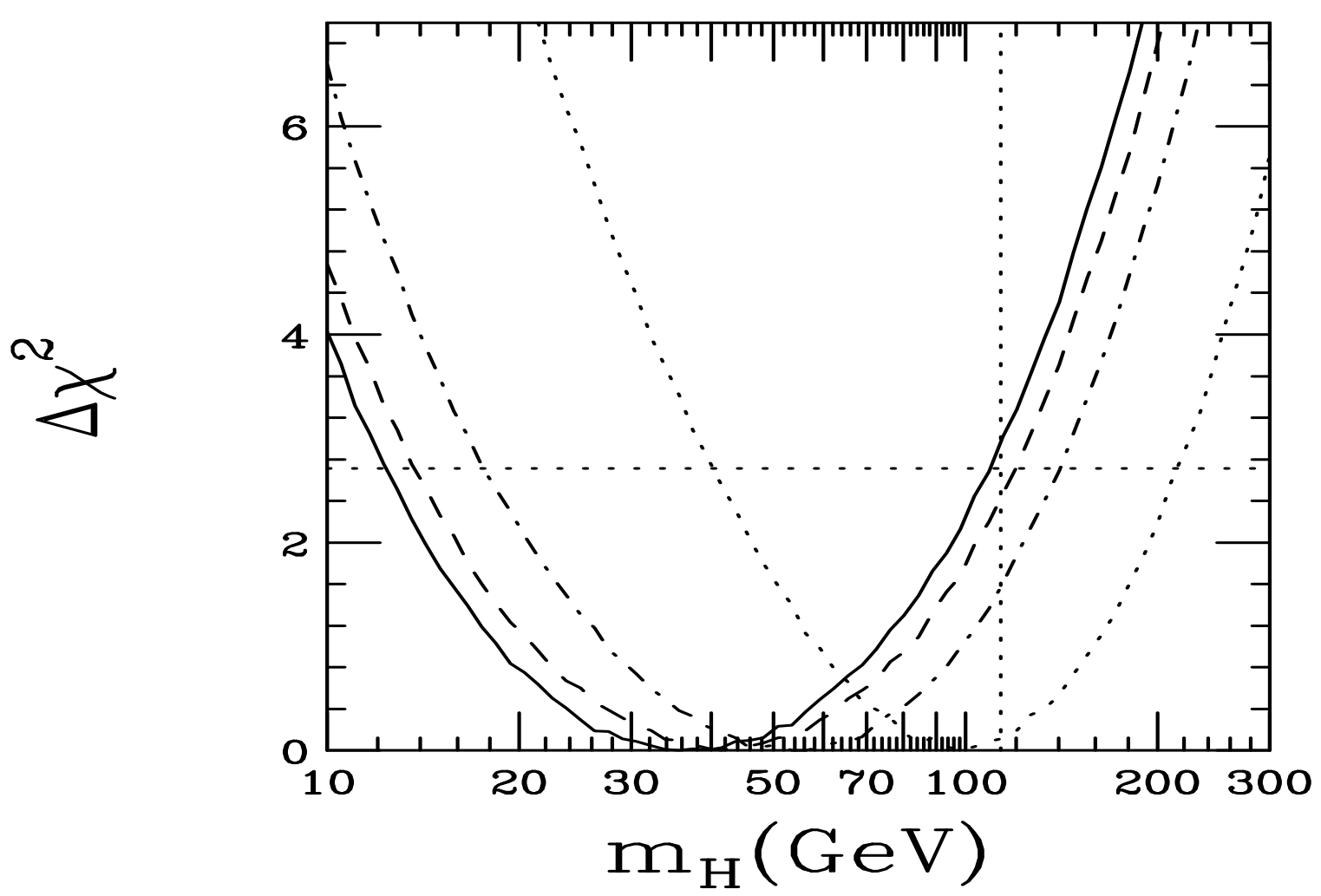

Figure 2: $\chi^{2}$ distributions as in figure 1. The lines correspond to fits of $m_{W}, \Gamma_{Z}$, and $R_{l}$, combined incrementally, as in table 2 , with the four leptonic asymmetry measurements (solid), plus $Q_{F B}$ (dashes), plus $A_{F B}^{c}$ (dot-dashes), plus $A_{F B}^{b}$ (dots). 